\title{
COVID-19 mortality rates for persons with intellectual and developmental disabilities
}

\author{
Mark D. Davis ${ }^{1}$, Scott Spreat ${ }^{2,}{ }^{*}$, Ryan Cox ${ }^{3}$, Matthew Holder ${ }^{4}$, Kathryn M. Burke ${ }^{5}$ and Donna M. Martin ${ }^{6}$ \\ ${ }^{1}$ Center for Disability Information Lemoyne, Pennsylvania, United States of American. \\ ${ }^{2}$ Research Institute at Woods Services Langhorne, Pennsylvania, United States of America. \\ ${ }^{3}$ New York Alliance for Inclusion and Innovation Albany, New York, United States of America. \\ ${ }^{4}$ Lee Specialty Clinic Louisville, Kentucky, United States of America. \\ ${ }^{5}$ Temple University Philadelphia, Pennsylvania, United States of America. \\ ${ }^{6}$ ANCOR Alexandria, Virginia, United States of America.
}

International Journal of Biological and Pharmaceutical Sciences Archive, 2021, 02(01), 179-183

Publication history: Received on 18 August 2021; revised on 23 September 2021; accepted on 25 September 2021

Article DOI: https://doi.org/10.53771/ijbpsa.2021.2.1.0075

\begin{abstract}
People with intellectual and developmental disabilities (IDD) appear to have an increased probability of death from COVID-19 once infected. We report infection and mortality rates for people with IDD compared to the general population of eight states at two time points during the COVID-19 pandemic. Note that these eight states contain approximately $1 / 3$ of the population of the United States. These data suggest individuals with IDD are less likely to be infected with the COVID-19 virus (5.62\%) than the general public (7.57\%). However, while mortality rates for both groups have declined over time, people with IDD are over twice as likely (2.29) to die from the infection as members of the general public.
\end{abstract}

Keywords: Intellectual Disability; COVID; Mortality: Infection

\section{Introduction}

During the early phase of the COVID-19 pandemic, multiple sources reported that people with intellectual and developmental disabilities (IDD) appeared to have an increased probability of death from a COVID-19 infection. Studying a large California database, Landes, Turk, and Wong [1] reported that individuals with intellectual disability have a fatality rate (once infected) 2.8 times greater than the comparable rate for members of the general public. In a sample including roughly one-third of the United States population, Spreat, Cox, \& Davis [2] reported a relatively similar factor of 1.8 times the rate of fatality for people with intellectual disability than the general public. Landes, Turk, Formica, McDonald, \& Stevens [3]. reported that COVID-19 fatality rates in New York group homes for people with intellectual disability were 1.9 times higher than the rates experienced by members of the general public. A recent national study covering January 2019 through November 2020 [4] reported that people with intellectual disability were more likely to become infected with COVID-19, more likely to become hospitalized, and more likely to die from COVID19 complications. The reported fatality rate was 5.9 times higher than that of the general public. It is likely that some of the variation in the reported fatality rates derives from sampling variations, but it is noteworthy that all published reports suggest that the COVID-19 fatality rate is markedly higher for people with IDD than it is for members of the general public. Researchers have advocated for the need for ongoing empirical research documenting disparities in COVID-19 effects for people with disabilities [5], and the early evidence outlined above indicates people with IDD are at critical risk for such disparities.

\footnotetext{
${ }^{*}$ Corresponding author: Scott Spreat

Research Institute at Woods Services Langhorne, Pennsylvania, United States of America. 
At the time of this research, COVID-19 pandemic continued, with the number of infections and the number of deaths growing daily. Data collected by Johns Hopkins University [6] suggest the probability of death resulting from a COVID19 infection for any individual has declined from almost 7\% in Spring of 2020 to slightly less than $2 \%$ in early 2021. Over one year after the pandemic onset in the United States in March 2020, vaccines have become available, and the medical field has developed means with which to reduce the probability of death from a COVID-19 infection. As such, these changes warrant an examination of the impact of COVID-19 on the health of people with IDD at both early (May 2020) and later points (January 2021) in the pandemic to examine how early trends related to infection and fatality rates have changed over time.

\section{Methods}

\subsection{Data Collection}

Eight states had provided COVID-19 mortality and infection data for an earlier study involving people with IDD [2]. These eight state authorities provided data as of January 31, 2021 (plus or minus four days) to the researchers through IDD provider associations within each state. The eight states were California, Colorado, Indiana, Maryland, New Jersey, New York, Pennsylvania, and Virginia. Data regarding infection and mortality trends within each participating state were obtained from the website maintained by Johns Hopkins University [6]:

\subsection{Study Participants}

The combined general population of these eight states was $107,722,117$, or roughly $33 \%$ of the estimated population of the United States as of July 2019. As of January 31, 2021, these eight states supported/served 614,330 adults who have IDD. The number of individuals with IDD being supported varied somewhat across the two points in time, but the magnitude of these differences was not significant $(0.5 \%)$.

\subsection{Data Analysis}

We compared rates of COVID-19 infection and fatality from COVID-19 once infected for people with IDD and the general public for the eight states in the sample at two separate points in time during the pandemic (May 2020 and January 2021).

\section{Results}

\subsection{Infection Rate}

As of May 31, 2020, 0.8\% of the general public had been diagnosed with a COVID-19 infection. At this same time, $0.9 \%$ of the individuals with IDD had received positive diagnoses. With the spread of the COVID-19 virus and the passage of eight months, the percentage of the population that had been infected had grown substantially. Approximately $7.57 \%$ of the general public and $5.62 \%$ of the individuals with IDD had been infected with the COVID-19 virus.

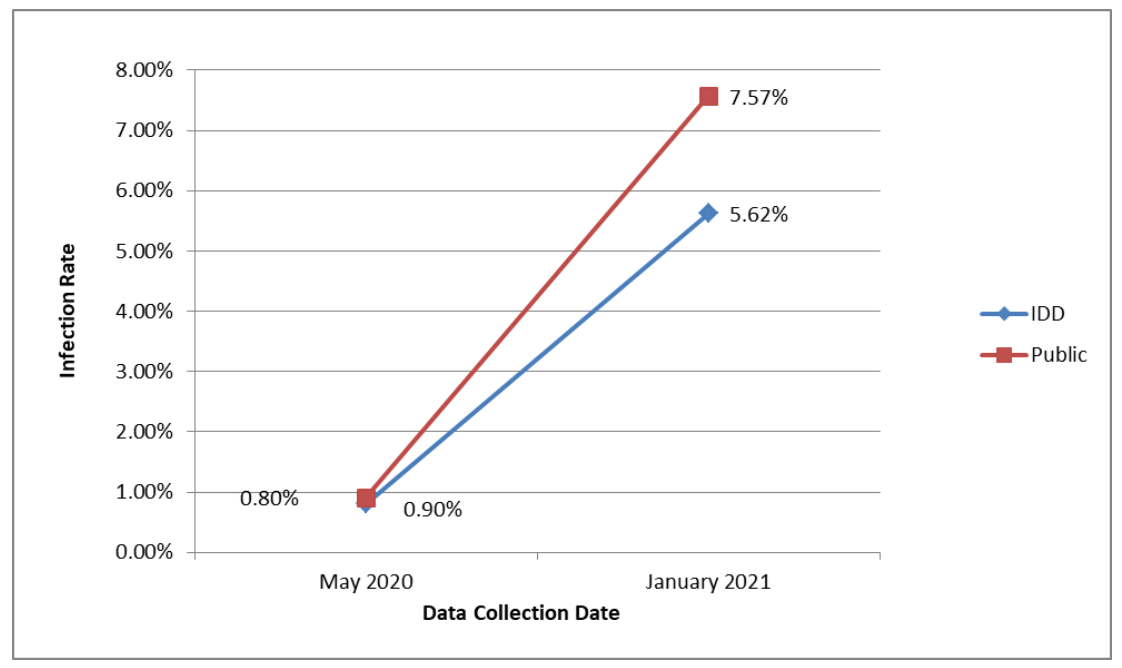

Figure 1 COVID-19 Infection Rates at Two Points in Time 
Figure 1 presents the infection rates at the two points in time. The infection rate for individuals with IDD was .74 times the infection rate for the general population.

\subsection{Fatality Rate}

The recent data from January 2021 are encouraging in that the fatality rates for both those with IDD and members of the general public have fallen. Once infected, the probability of death from COVID-19 infection was less than $2 \%$ (1.94\%) for members of the general public and under $5 \%(4.41 \%)$ for individuals with IDD. While the fatality rate has declined for both people with IDD (64\%) and the general public (71\%), the disproportionate impact on people with IDD has remained. Both members of the general public and people with IDD are now less likely to die once infected, but people with IDD are still at least twice as likely (2.29) to die from the infection as are members of the general public. The decline and the continuing disparity are represented in Figure 2.

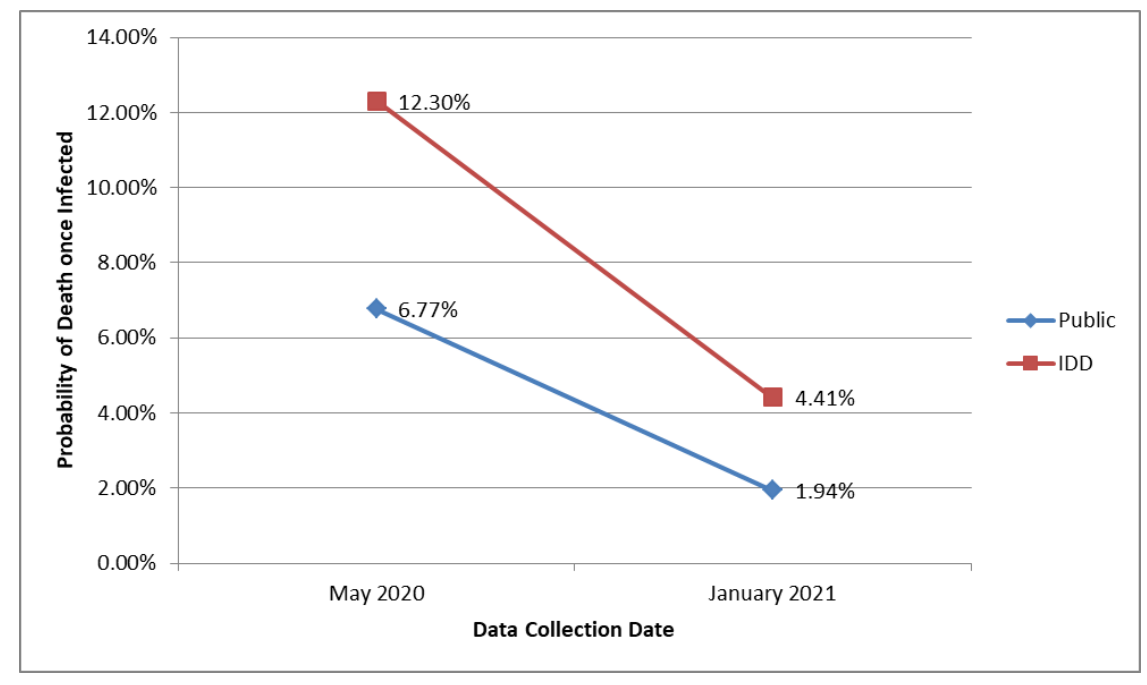

Figure 2 Probability of Death Once Infected at Two Points in Time

\section{Discussion}

It is heartening to learn that the fatality rate for both people with IDD and members of the general public declined dramatically over the first eight months of the pandemic. The probability of death, once infected with COVID-19, overall declined over 70\%. Despite this relatively positive outcome, it is noted that people with IDD appear to have retained an increased probability of COVID-19 death when compared with members of the general public.

It has been hypothesized that the increased fatality rate for people with IDD is largely a function of health issues that are commonly co-morbid with IDD. Turk and McDermott [7] noted that people with IDD tend to have a higher number of health issues than do members of the general public, and that the number of co-morbid conditions was a major predictor of COVID-19 fatality. Supporting this observation, researchers in Ireland reported, after reviewing records of 66 individuals with intellectual disability who died from COVID-19, that these individuals had high levels of epilepsy, dysphagia, mental illness, dementia, and tended to be overrepresented in lower functioning ranges of intellectual disability [8]. In discussing the increased COVID-19 fatality rate for people with Down Syndrome, Clift, Coupland, Keogh, Hemingway, \& Hippisley-Cox [9]. reported that their analysis suggested that cardiovascular and pulmonary challenges were at least partially responsible for the increased mortality rate. Despite the credibility of the hypotheses regarding comorbid conditions, Gleason, Ross, Fossi, Blonsky, Tobias, \& Stephens [4] noted that even when comorbid conditions were statistically controlled, the presence of intellectual disability was the largest predictor of negative outcome. Clearly, additional research is warranted regarding the impact of co-morbidity on COVID-19 related deaths.

It was also noted in the present study that individuals with IDD were slightly less likely to become infected by the COVID19 virus, particularly at the second time point. The modest difference is perhaps attributable to the closing of many day programs, the general limitation on community-based activities. While subject to empirical verification, it may be that the IDD provider community, despite the regular rotation of staff into homes, was better able to implement COVID-19 mitigation strategies, such as masking and social separation than were members of the general public. It was early understood that people with intellectual and developmental disabilities were inherently at higher risk of poor COVID- 
19 outcomes, confirmed by all early empirical evidence [1,2,3,4]. This finding is a very positive testament to the responsiveness of agencies to the pandemic, once resources became available for them to mitigate risks.

\section{Limitations and Directions for Future Research}

It should be noted that the collected data were not submitted to statistical analysis beyond simple reportage of summary figures. With a large sample such as was employed here, any observed difference would have achieved statistical significance. The issue must instead shift to the matter of practical significance. For example, the difference in infection rate in May 2020 achieved statistical significance in trial runs via chi-square analysis, but one must evaluate the realworld impact of the $0.1 \%$ difference. On the other hand, the roughly $2 \%$ difference noted in January 2021 suggests that almost 12,000 more individuals with IDD would have been infected with COVID-19 if matters were equal. And, based on the fatality data, we would estimate that of those individuals, over 500 would have died. We suggest additional research on a longitudinal basis would be helpful to discern the etiology for the increased mortality risk for people with IDD.

It must be recognized that this was a population-based study, incorporating data on people with and without IDD in eight states. Additionally, it is likely that many factors beyond the mere presence of IDD distinguish these two groups, and that factors other than the classification of IDD may explain the observed outcomes. For example, it is noted that people with intellectual disability tend to have slightly shorter life spans than the general public [10], so it is reasonable to suggest that age may be a distinguishing factor. An age matched cohort study would shed additional light on this question.

\section{Conclusion}

These data suggest that people with intellectual and developmental disabilities were 2.29 times more likely to die from a COVID-19 infection, but only .74 times as likely to become infected. Conjointly, consideration of both infection rate and mortality rate would suggest that people with IDD are roughly 1.7 times as likely to die from COVID-19 when mitigation strategies are employed. The point is that industry-wide mitigation efforts were more effective than general societal efforts, and this helped to offset some of the increased medical risk associated with the category of IDD.

It should be noted that there are hundreds of causes of IDD. Some of these are genetic syndromes, like Down syndrome, that may specifically place individuals at higher risk due to poorly understood, genetically-related mechanisms that are unique to the syndrome and do not apply to the rest of the IDD population. It is also likely that there are some high risk factors that are more common in the IDD population, compared to the neurotypical population. In order to be truly responsive to the needs of the IDD population, these observations suggests that next research steps should focus on the identification of major driving factors of susceptibility to COVID-19 infection and death.

\section{Compliance with ethical standards}

\section{Acknowledgments}

The authors thank and acknowledge the contribution of the eight IDD provider associations whose acquisition of data form their state authorities permitted this analysis.

\section{Disclosure of conflict of interest}

None of the authors report any conflict of interest in the conduct of this study. The study was not a funded project.

\section{References}

[1] Landes S, Turk M, Wong A. Covid-19 outcomes among people with intellectual and developmental disabilities in California. Disability and Health Journal. 2021; 14(2): 101051.

[2] Spreat S, Cox R, Davis M. Covid-19 case and mortality report: Intellectual or developmental disabilities. Lemoyne,PA:PAR. 2020.

[3] Landes S, Turk M, Formica M, McDonald K, Stevens J. COVID-19 outcomes among people with intellectual and developmental disability living in residential group homes in New York state. Disability and Health Journal. 2020; $13(4)$. 
[4] Gleason J, Ross W, Fossi A, Blonsky H, Tobias J, Stephens M. The devastating impact of COVID-19 on individuals with intellectual disabilities in the United States. New England Journal of Medicine Catalyst. 2021; 2(2).

[5] Turk M, Mitra M. People with disability and the COVID-19 pandemic: The needforempiric research. Disability and Health Journal. 2021; 14(2).

[6] Johns Hopkins University. Mortality analyses. Coronavirus Resource Center. 2021: Baltimore, Maryland, United States.

[7] Turk M, McDermott S. The COVID-19 pandemic and people with disability. Disability and Health Journal. 2020; 13(3).

[8] Perera B, Laugharne R, Henley W, Zabel A, Lamb K, Branford D, Courtanay K, Alexander R, Purandare K, Wijeratne A, Radhakrishnan V, McNamara E, Daureeawoo Y, Sawhney I, Scheepers M, Taylor G, Shankar R. COVID-19 deaths in people with intellectual disability in the UK and Ireland: Descriptive study. British Journal of Psychiatry Open. $2020 ; 16(6)$

[9] Clift A, Coupland C, Keogh R, Hemingway H, Hippisley-Cox,J. COVID-19 mortality risk in Down syndrome: Results from a cohort study of 8 million adults. Annals of Internal Medicine. 2020.

[10] Copus A. People with intellectual disability: What do we know about adulthood and life expenctancy? Developmental Disabilities Research Reviews. 2013; 18(1): 6-16. 